\title{
Dielectric properties of ceramic materials obtained from rice husk for electronic applications
}

\begin{abstract}
This paper reports on dielectric properties of ceramic material obtained from Rice Husk $(\mathrm{RH})$ for electronic applications. $\mathrm{RH}$ is considered as agriculture waste material, contains 20$25 \mathrm{wt} \%$ of silica ( $\mathrm{SiO} 2)$. The nanometer-sized silica powder was prepared via mechanical alloying and subsequent molding into pellet form. The compacted pellet samples were sintered at $800^{\circ} \mathrm{C}, 1000^{\circ} \mathrm{C}, 1100^{\circ} \mathrm{C}$ and $1200{ }^{\circ} \mathrm{C}$ respectively. Silver paste was applied on both faces of the pellet to act as electrodes. For analysis of ferroelectric polarization with respect to electric field, measurement was done using ferroelectric hysteresis loop towards the sintered pellets. The dielectric analysis was done using LCR meter. It clearly shows that dielectric constant of the samples decrease with increasing applied frequency. The capacitance is found to be decreasing with increased sintering temperatures. The result showed that WHRA does contain silica which has high dielectric constant and therefore is suitable for electronics application.
\end{abstract}

Keyword: Rice husk silica; Dielectric; Capacitance; Hysteresis loop 